\title{
Pain assessment and management of trauma patients in an emergency department of a tertiary hospital in Tanzania
}

George D. Dilunga ${ }^{1}$, Hendry R. Sawe ${ }^{1,2^{*}}$ (D, Irene B. Kulola ${ }^{1,3}$, Juma A. Mfinanga ${ }^{1}$, Nanyori J. Lucumay', Elly M. Mulesi ${ }^{1}$ and Ellen J. Weber ${ }^{1}$

\begin{abstract}
Background: Proper pain assessment is a core component in management of trauma patients but prior literature has suggested that pain management is inadequate in emergency settings. With the development of emergency medicine in low-income countries (LIC), the procedures for pain assessment and management of trauma patients have not been well studied and protocols have not been established. We aimed to describe practices of pain assessment and management in an emergency department in Tanzania.
\end{abstract}

Methods: This was a prospective cohort study of consecutive adult trauma patients presenting to the Emergency Medicine Department of Muhimbili National Hospital (EMD-MNH) in Dar es Salaam, Tanzania, from July 2017 to December 2017. A case report form (CRF) was used to record demographics and clinical characteristics of participants, whether or not pain was assessed at either triage or in the treatment area, and the administration of pain medications. The assistant also assessed pain independently with the numeric rating scale (NRS) of (0-10). Outcomes were proportions of patients who received pain assessment, patients who received pain medication, and types of medications administered. Descriptive data is summarised using frequency, percentage, and median with interquartile ranges as appropriate. Chi-square tests were used to determine association between pain assessments, receipt of pain medication, and types of medications.

Results: We enrolled 311 (10.9\%) trauma patients during the period of study. The median age was 32 years (IQR 25-43 years), and 228 (73.3\%) were male. The most common mechanism of injury was motor vehicle crash 185 (59.4\%), and of these, 87 (47\%) involved motorcycles. Three hundred ten (99.6\%) patients had pain assessment documented arrival, and 285 (91.6\%) had a second assessment. Pain scores obtained by the research assistant were as follows: mild pain score (NRS 1-3) 154 (49.5\%) patients, moderate pain (NRS 4-6) 68 (21.8\%), and severe pain (NRS 7-10) 89 (28.7\%). Pain medications were given to 144 (46.3\%) patients, 29 (20.1\%) of those with mild pain, 41 (28.7\%) of those with moderate pain score, and 74 (51.4\%) of those with severe pain. The use of opiates increased with increased pain severity.

Conclusions: In this ED in LIC, the assessment of pain was well documented; however, less than half of patients with documented pain received pain medication while at the ED. Future studies should focus on identification of factors affecting the provision of pain medications to trauma patients in the ED.

Keywords: Pain assessment, Pain management, Trauma, And tertiary hospital

\footnotetext{
* Correspondence: hendry_sawe@yahoo.com

'Emergency Medicine Department, Muhimbili University of Health and Allied

Science, P.O. Box 65001, Dar es salaam, Tanzania

${ }^{2}$ Emergency Medicine Department, Muhimbili National Hospital, Dar es

Salaam, Tanzania

Full list of author information is available at the end of the article
}

(c) The Author(s). 2018 Open Access This article is distributed under the terms of the Creative Commons Attribution 4.0 International License (http://creativecommons.org/licenses/by/4.0/), which permits unrestricted use, distribution, and reproduction in any medium, provided you give appropriate credit to the original author(s) and the source, provide a link to the Creative Commons license, and indicate if changes were made. The Creative Commons Public Domain Dedication waiver (http://creativecommons.org/publicdomain/zero/1.0/) applies to the data made available in this article, unless otherwise stated. 


\section{Background}

Trauma is among the leading cause of morbidity and mortality around the globe with the highest frequency in low-income countries (LICs) [1]. Pain is a prominent feature among trauma patients presenting at the EDs worldwide. Pain assessment and management is a crucial aspect in management of patients presenting at the emergency departments. Better pain management leads to improved satisfaction of patients as well as decreased length of stay in hospital [2].

Several pain assessment tools have been developed over time. When these tools are used, they have proven to be helpful in helping the patients in their management of their pain [3]. There is also a pain assessment and documentation tool (PADT) that has been developed in order to better understand the effectiveness of treatment given to patients in pain [4].

In high-income countries, there is still improper assessment and management of pain in trauma patients. Pain relief is still more "rhetoric than a reality" despite the advancements in acute pain teams [5]. In low- and middle-income countries, the management of pain in acute care settings has not been well studied. This is particularly true in Sub Saharan Africa (SSA), where emergency medicine is still a developing speciality. Anecdotally, many acute receiving areas use subjective assessment to determine levels of pain $[6,7]$.

Tanzania opened its first full-capacity emergency department, staffed by emergency medicine specialists in 2010 [8]. We aimed to describe the burden of traumatic pain, frequency of its assessment, and subsequent management among patients presenting with trauma to this hospital's ED. The information extrapolated from this study will help us determine how well pain is managed and lay a foundation for protocols for management of acute traumatic pain.

\section{Methods}

\section{Study design}

This was a prospective, descriptive study of consecutive adult trauma patients presenting to Muhimbili National Hospital Emergency Medicine Department (MNH-EMD) between July 2017 and December 2017.

\section{Study setting}

The MNH-EMD is located in Dar es Salaam, the business capital of Tanzania. It is part of a tertiary referral hospital with a total bed capacity of about 1500 [9]. The ED is at the forefront of the hospital and most of the patients coming into the hospital pass through it.

The ED sees about 1200 patients per week. The top five most commonly occurring complaints at the ED include trauma, infections, mental health cases, neoplasm, and issues relating to pregnancy [8]. Most admitted trauma patients are referred to Muhimibili Orthopedic Institute where orthopaedics and neurosurgery are housed.

\section{Participants}

Adult patients 18 years of age and above who presented with the complaint of pain as a result of trauma to the EMD were eligible. Patients were excluded if they were haemodynamically unstable with $\mathrm{SBP}<90 \mathrm{mmHg}$ or altered mental status GCS $<15$.

\section{Procedures}

A research assistant was present in the ED at various times during the study period, both days and nights, and enrolled a convenience sample of patients meeting the inclusion criteria and who consented. Patients were approached in the treatment areas, after triage. Using a case report form (CRF) (Additional file 1), the research assistant collected information on the patient's demographics, clinical presentation, whether or not pain was assessed, administration of pain medication, ED diagnoses, and disposition of based on both patient's enrolled patients interview and review of the electronic medical record (WELLSOFT Version 11 Corporation, Somerset,

Table 1 Characteristics of study patients

\begin{tabular}{|c|c|c|}
\hline Demographics & $\begin{array}{l}\text { Number } \\
(N)\end{array}$ & $\begin{array}{l}\text { Percentage } \\
(\%)\end{array}$ \\
\hline \multicolumn{3}{|l|}{ Sex } \\
\hline Male & 227 & 73 \\
\hline Female & 84 & 27 \\
\hline \multicolumn{3}{|l|}{ Age group } \\
\hline $18-30$ years & 139 & 44.7 \\
\hline $31-45$ years & 106 & 34.1 \\
\hline $46-60$ years & 43 & 13.8 \\
\hline$>60$ years & 23 & 7.4 \\
\hline \multicolumn{3}{|l|}{ Referral status } \\
\hline Referred & 217 & 69.7 \\
\hline Self-referral & 94 & 30.2 \\
\hline \multicolumn{3}{|l|}{ NRS pain score } \\
\hline Mild (1-3) & 154 & 49.5 \\
\hline Moderate (4-6) & 68 & 21.8 \\
\hline Severe $(7-10)$ & 89 & 28.7 \\
\hline \multicolumn{3}{|l|}{ Mechanism of injury } \\
\hline Motor vehicle crash & 185 & 59.5 \\
\hline Fall & 63 & 20.3 \\
\hline Assault & 37 & 11.9 \\
\hline Burn & 9 & 2.9 \\
\hline Gunshot wound & 8 & 2.6 \\
\hline Animal bite & 7 & 2.3 \\
\hline Stab wound & 2 & 0.6 \\
\hline
\end{tabular}


NJ, USA). The research assistant independently assessed pain for each patient using the validated numerical rating scale (NRS) and recorded it on the form. The treating physician was not told of the result on the NRS.

\section{Outcomes}

Primary outcome of the study was the proportion of all patients who has a pain assessment performed (at triage and/or the treatment room) and proportion who received pain medication. Secondary outcomes were the administration of pain medication according to the NRS level and the class of pain medication used.

\section{Data analysis}

Sample size estimate was based on the proportion of patients who received pain assessment (54\%) in a study done in Ouagadougou [10]. To achieve a 95\% confidence interval with a width of $10 \%$, a minimum number of 382 patients would be required.

The data from the CRF was transferred into Excel spreadsheet (Microsoft Corporation, Redmond, WA, USA) and then transferred to SPSS and analysed. Median with interquartile ranges and percentages were calculated for descriptive data. Chi-square tests were used to determine relationship between NRS scores and receipt of pain medication, and association of severity of pain with pain assessment, and severity of pain with type of pain medication.

\section{Results}

During the study period, 2848 trauma patients presented at the ED. We enrolled 311 patients. Of the 311 patients enrolled, median age was 32 years (IQR 25-43 years) and 227 (73\%) were male. The majority 217 (69.7\%) were referred from other hospitals. The most common identified mechanism of injury was motor vehicle crash in 185 (59.4\%) of patients Table 1.

\section{Pain management in the ED}

Of the 311 patients enrolled in the study, 310 (99.6\%) received pain assessment initially (Fig. 1). Of these, 285 (91.6\%) had pain reassessment done to them. One patient did not receive pain assessment at any point in the ED. Pain medications were given to $144(46.3 \%)$ of the patients.

Pain assessment, NRS scores, and pain management Overall, 285/311 (91.6\%) patients received pain assessment by EMD Provider on arrival (Fig. 1). Among those who received assessment, $149(52.2 \%)$ had mild pain, 59 (20.7\%) had moderate pain, and (27.0\%) had severe pain. Overall, pain medications were given to 144 (46.3\%) of patients. The research assistant (RA) independently conducted an assessment of all 311 patients using the NRS. The NRS pain scores were mild (1-3) 154 (49.5\%), moderate (4-6) 68 (21.8\%), and severe (7-10) 89 (28.6\%). Pain medication was given to $29 / 154$ (18.8\%) patients

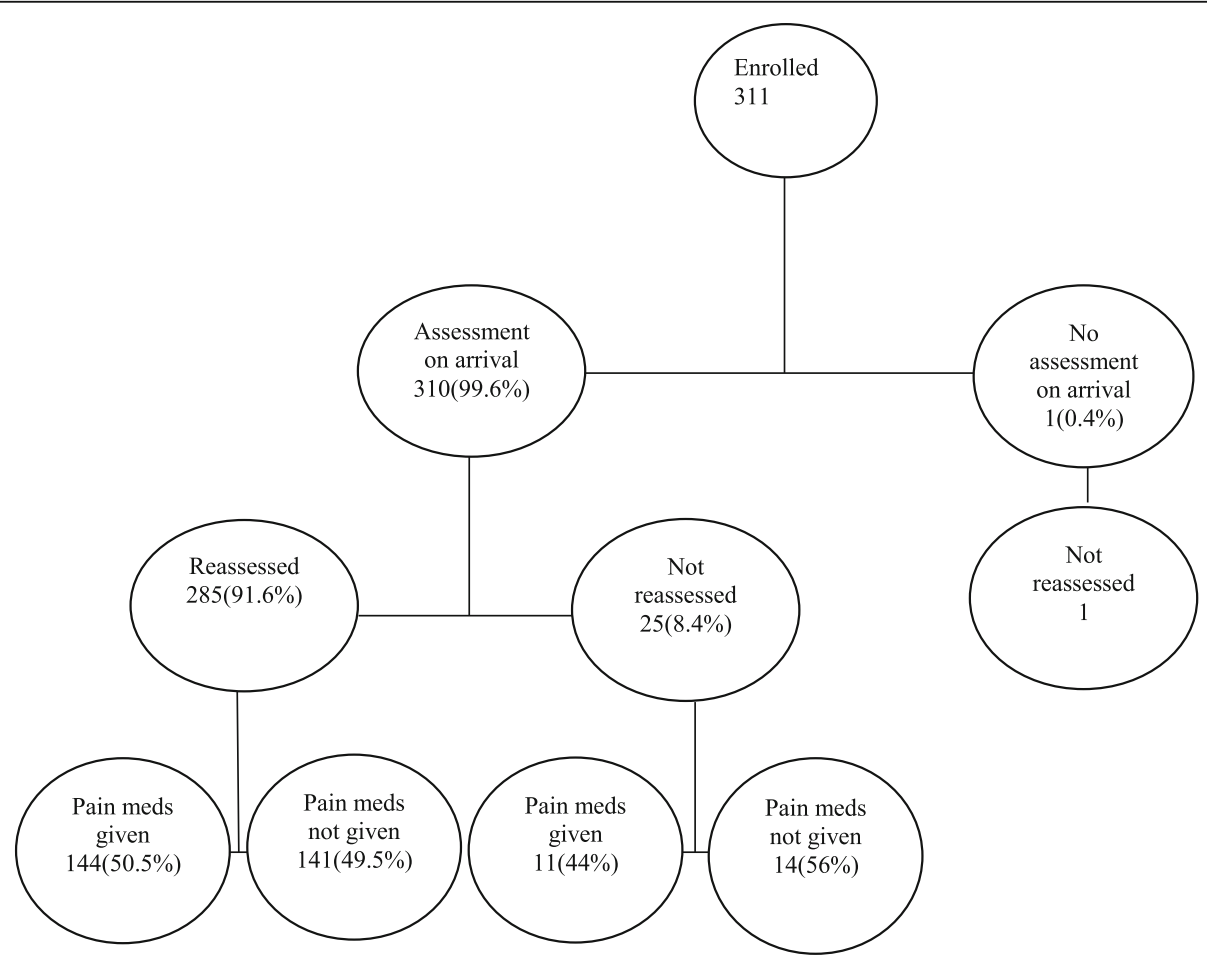

Fig. 1 Pain assessment and management of study participants in the emergency department 
Table 2 Pain assessment and management of adult trauma patients by pain level

\begin{tabular}{lllll}
\hline NRS pain score & Mild & Moderate & Severe & $P$ value \\
\hline Provider pain assessment $\left({ }^{a} N=285\right)$ & $149(52.2 \%)$ & $59(20.7 \%)$ & $77(30.1 \%)$ & 0.008 \\
RA NRS pain assessment $\left({ }^{b} N=311\right)$ & $154(49.5 \%)$ & $68(21.8 \%)$ & $89(28.6 \%)$ & $<0.00001$ \\
Pain medications given in EMD $(n / N)$ & $29 / 154(18.8 \%)$ & $41 / 68(60.3 \%)$ & $74 / 89(83.1 \%)$ & $<0.00001$
\end{tabular}

26 patients did not receive pain reassessment

${ }^{\text {b }}$ Research assistant (RA) performed NRS pain assessment

with mild pain, 41/68 (60.3\%) with moderate pain, and $74 / 89$ (83.1\%) with severe pain. Patients with more severe pain on the NRS were more likely to receive pain medications $(p<0.00001)$ Table 2.

\section{Types of pain medications according to pain scores}

Opioids were the most prescribed medications given to $97(68 \%)$ out of 144 patients who received pain medications. Patients with severe pain were more likely to receive opioids than those with lower pain scores, whereas patients with mild pain were more likely to receive acetaminophen or non-steroidal anti-inflammatory medications Table 3.

\section{Discussion}

Pain assessment has been shown to improve the outcomes of patients who present to the ED with pain as a result of trauma [2, 3]. In our study, we found that nearly all patients received pain assessment on arrival, and most were reassessed. However, only half of the patients received pain medications.

The frequency of pain assessment found in our study is higher than in most studies in other parts of the world. In a study in Morocco, only $14 \%$ of the patients were assessed for pain while in a study in Ouagadougou about $54 \%$ of the trauma patients received pain assessment $[10,11]$. A study in Norway found that $77 \%$ of patients were evaluated for pain on arrival at the ED [5]. In a multi-centre study in the USA and Canada, $83 \%$ of patients with pain intensity of 4 or more received pain assessment, and $31 \%$ had a repeat assessment [12].

This suggests that pain has become the "fifth vital sign" in the developing specialty of emergency medicine in Tanzania [13]. However, there were some patients who had a high pain score as determined by the research assistant but did not receive pain assessment by providers in the treatment and resuscitation rooms. This could have been due to their severity of pain that

Table 3 Types of pain medications according to pain scores

\begin{tabular}{lllll}
\hline NRS pain Score & Mild & Moderate & $\begin{array}{l}\text { Severe } \\
N=74\end{array}$ & $P$ value \\
& $N=29$ & $N=41$ & $N=1 \%$ & 0.0001 \\
Opioids & $3(3.1 \%)$ & $25(60.9 \%)$ & $69(93.2 \%)$ & 0.003 \\
Acetaminophen & $7(24.1 \%)$ & $4(9.7 \%)$ & $2(2.7 \%)$ & 0.0001 \\
NSAIDs & $19(65.5 \%)$ & $12(29.3 \%)$ & $3(4.1 \%)$ & 0.000 \\
\hline
\end{tabular}

prompted providers to manage the pain without documentation of pain assessment.

Muhimbili National hospital is a tertiary referral hospital that receives patients from various parts of the country [9]. Of patients, 70\% were referred and may have been given pain medication prior to arrival, resulting in overall lower pain levels. Nevertheless, half of the patients had moderate or severe pain. In most previous studies in HICs as well as LMICs, most patients had moderate to severe pain scores [4] (Fig. 2).

In our study, $144(46.3 \%)$ of trauma patients received pain medications. The percentages of those receiving pain medications increased with increasing pain score while the percentages of those not receiving pain medications decreased with increasing pain score. This tallies with the WHO recommendation of pain management which involves the provision of pain medications to patients with the score of 6 or higher [14]. Importantly, less than half of all patients who had pain assessment documented received any pain medications. Some patients who had low pain scores still received pain medications while some who had a high pain score did not receive pain medications. While we did not inquire exact reasons for low rate of provision of anti-pain medications, we believe number of factors might contribute towards this, including access to medications, underassessment, and poor documentation.

The type of medication given in general varied according to the degree of pain. Opioids were given more frequently to patients with a higher pain score compared to those with mild pain score. Less strong medications for pain like acetaminophen were given to patients with lower pain scores compared to those with higher pain scores. In a previous study done at Muhimbili Orthopaedic Institute, opioids were not used at all to treat severe pain in long bone fractures [15]. Thus, our findings suggest improved compliance with recommendations of the World Health Organization on management of pain [16].

Although we saw a greater level of assessment than in many other studies, our study shows similar problems with regard to delivery of pain medication. The reasons for receiving pain medications and the barriers for not receiving pain medications at Muhimbili may however be different compared to HICs [17]. Patients with low pain scores may have sometimes asked for pain 


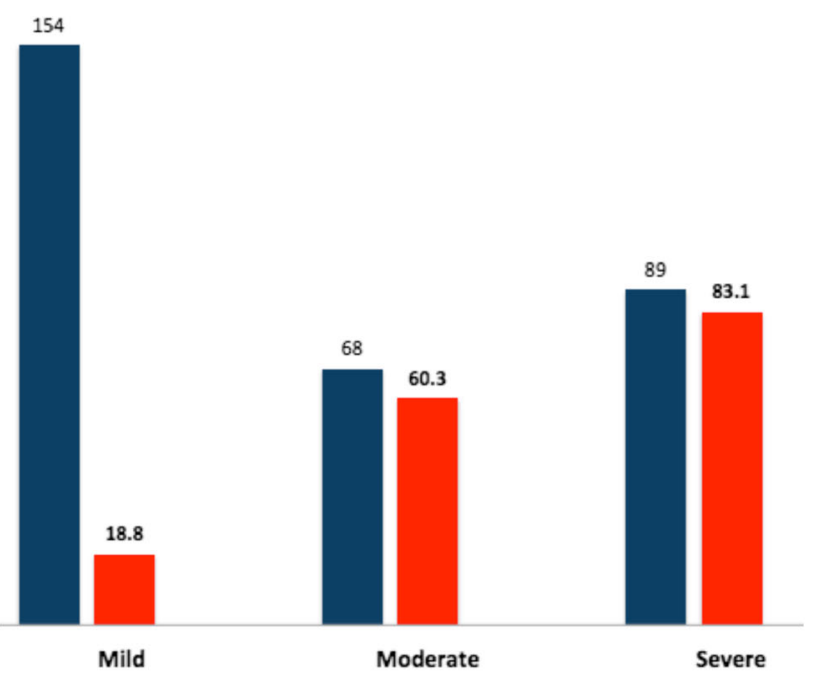

Fig. 2 Plot of pain level and provision of pain medication at the EMD

treatment while some with high pain scores might have refused pain treatment. Certain pain medications are sometimes not available. Therefore, further studies are needed in order to identify the reasons for lack of provision of medications to trauma patients in the ED and also create a clear protocol in our setting.

\section{Limitations}

The major limitation in our study was that this was a single-site descriptive study over a short duration of time. We did not have seasonal variations of trauma patients especially the ones who present during the busy travelling and holiday seasons. Therefore, our findings may not necessarily reflect the general picture of a busy ED. Due to the limited nature of the study time, we did collect the required sample size and not all patients with trauma were enrolled in the study, and thus, it is not clear that the patients are representative. However, the characteristics of our patients are similar to those in other studies [7, 18, 19]. We collected NRS pain scores and used these to compare treatment with scores; it is possible the locally used system resulted in different responses. However, we did not use this as it is not a standardised scale with external validity.

\section{Conclusion}

The assessment of pain in our setting was well documented in contrast with findings of prior studies in HICs. However, less than half of patients with documented pain received pain medications while at the EMD. Our study lays a foundation for upcoming studies focusing on identification of factors affecting the provision of pain medications to trauma patients and improving management of pain in these patients.

\section{Additional file}

Additional file 1: S1. Case report form (CRF). (PDF $153 \mathrm{~kb}$ )

\section{Acknowledgements}

The author would like to thank Prof. Mwafongo, Dr. Said Kilindimo, Dr. Upendo George, and Dr. Bernard Kepha for their unwavering support, also to the research assistants and all who made the study possible.

\section{Funding}

This was a non-funded project. The principal investigators used their own funds for logistics, data collection, and analysis.

Availability of data and materials

The datasets used and/or analysed during the current study are available from the corresponding author on request.

\section{Author's contributions}

GDD contributed to the conceptualization, data curation, formal analysis, funding acquisition, methodology, project administration, validation, writing the original draft and writing review, and editing. HRS was involved in the conceptualization, data curation, formal analysis, methodology, and supervision as well as in the revision of the manuscript and writing review and editing. IBK contributed to the design, formal analysis, supervision, writing review, and editing as well as to the review of the manuscript. JAM contributed design, formal analysis, supervision, writing review and editing as well as review of manuscript. NJL contributed to the design, formal analysis, supervision, writing review, and editing as well as to the review of manuscript, EMM contributed to the conceptualization and analysis, as wel as to the review of manuscript. EJW contributed to the conceptualization, data curation, formal analysis, methodology, supervision, validation, and critical review of the manuscript. All authors read and approved the final manuscript.

\section{Ethics approval and consent to participate}

The study protocol was reviewed and approved by the Institutional Review Board of the Muhimbili University of Health and Allied Sciences and permission to conduct the study was obtained from Muhimbili National Hospital administration. All patients enrolled provided written consent.

Consent for publication

Not applicable

Competing interests

The authors declare that they have no competing interests. 


\section{Publisher's Note}

Springer Nature remains neutral with regard to jurisdictional claims in published maps and institutional affiliations.

\section{Author details}

${ }^{1}$ Emergency Medicine Department, Muhimbili University of Health and Allied Science, P.O. Box 65001, Dar es salaam, Tanzania. ${ }^{2}$ Emergency Medicine Department, Muhimbili National Hospital, Dar es Salaam, Tanzania. ${ }^{3}$ Department of Emergency Medicine, University California San Francisco, San Francisco, CA, USA.

Received: 20 August 2018 Accepted: 25 October 2018

Published online: 07 November 2018

\section{Referrences}

1. Addressing the Growing Burden of Trauma and Injury in Low- and MiddleIncome Countries | AJPH | Vol. 95 Issue 1 [Internet]. [cited 2017 Jun 9]. Available from: http://ajph.aphapublications.org/doi/abs/10.2105/AJPH.2004. 039354

2. Silka PA, Roth MM, Moreno G, Merrill L, Geiderman JM. Pain scores improve analgesic administration patterns for trauma patients in the emergency department. Acad Emerg Med. 2004;11(3):264-70.

3. Assessment of pain | BJA: British Journal of Anaesthesia | Oxford Academic [Internet]. [cited 2017 Jun 9]. Available from: https://academic.oup.com/bja/ article-lookup/doi/10.1093/bja/aen103

4. Singer AJ, Garra G, Chohan JK, Dalmedo C, Thode HC. Triage pain scores and the desire for and use of analgesics. Ann Emerg Med. 2008;52(6):689-95.

5. Dale J, Bjørnsen LP. Assessment of pain in a Norwegian Emergency Department. Scand J Trauma Resusc Emerg Med. [Internet]. 2015 Oct 29 [cited 2017 Feb 17];23. Available from: http://www.ncbi.nlm.nih.gov/pmc/ articles/PMC4625614/.

6. Awolola AM, Campbell L, Ross A. Pain management in patients with longbone fractures in a district hospital in KwaZulu-Natal South Africa. Afr J Prim Health Care Fam Med. 2015;7(1):818.

7. Size M, Soyannwo OA, Justins DM. Pain management in developing countries. Anaesthesia. 2007;62:38-43.

8. Muhimbili Emergency Medicine Program [Internet]. [cited 2015 Jan 16]. Available from: https://emd.muhas.ac.tz.

9. Our Profile - MUHIMBILI NATIONAL HOSPITAL [Internet]. [cited 2015 Jan 16]. Available from: http://www.mnh.or.tz/index.php/our-profile.

10. Assessment of the Care of Acute Pain at the Trauma Center of Ouagadougou [Internet]. [cited 2017 Feb 17]. Available from: https:/www. jscimedcentral.com/Anesthesiology/anesthesiology-1-1016.php

11. Damghi $\mathrm{N}$, et al. Patient satisfaction in a Moroccan emergency department. Int Arch Med. 2013;6:20.

12. Paula Tanabe KHT. Pain in the Emergency Department: results of the Pain and Emergency Medicine Initiative (PEMI) multicenter study. J Pain. 2007; 8(6):460-6.

13. Kelly A-M. A process approach to improving pain management in the emergency department: development and evaluation. J Accid Emerg Med. 2000;17(3):185-7.

14. Stephan F-P, Nickel CH, Martin JS, Grether D, Delport-Lehnen K, Bingisser R. Pain in the emergency department: adherence to an implemented treatment protocol. Swiss Med Wkly. 2010;140(23-24):341-7.

15. Haonga BT, Makupa JE, Muhina Rl, Nungu KS. Pain management among adult patients with fractures of long bones at Muhimbili Orthopaedic Institute in Dar es Salaam Tanzania Tanzan. J Health Res. 2011;13(4):107-11.

16. Undertreatment of acute pain (oligoanalgesia) and medical practice variation in prehospital analgesia of adult trauma patients: a $10 \mathrm{yr}$. retrospecti... - PubMed - NCBI [Internet]. [cited 2017 Feb 17]. Available from: https://www.ncbi.nlm.nih.gov/pubmed/23059961

17. Patrick PA, Rosenthal BM, lezzi CA, Brand DA. Timely pain management in the emergency department. J Emerg Med. 2015;48(3):267-73.

18. Herr $\mathrm{K}$, Titler M. Acute pain assessment and pharmacological management practices for the older adult with a hip fracture: review of ED trends. J Emerg Nurs. 2009;35(4):312-20.

19. Huang KTL, Owino C, Gramelspacher GP, Monahan PO, Tabbey R, Hagembe $M$, et al. Prevalence and correlates of pain and pain treatment in a western Kenya referral hospital. J Palliat Med. 2013;16(10):1260-7.

\section{Ready to submit your research? Choose BMC and benefit from:}

- fast, convenient online submission

- thorough peer review by experienced researchers in your field

- rapid publication on acceptance

- support for research data, including large and complex data types

- gold Open Access which fosters wider collaboration and increased citations

- maximum visibility for your research: over $100 \mathrm{M}$ website views per year

At $\mathrm{BMC}$, research is always in progress.

Learn more biomedcentral.com/submissions 